\title{
Calgranulin A (S100A8) Immunostaining: A Future Candidate for Risk Assessment in Patients with Non-Muscle-Invasive Bladder Cancer (NMIBC)
}

\author{
André P. Nicklas · Mario W. Kramer · Jürgen Serth · Jörg Hennenlotter • \\ Marie C. Hupe - Daniel U. Reimer - Arnulf Stenzl • Axel S. Merseburger • \\ Markus A. Kuczyk · Christoph-Alexander J. von Klot
}

Received: June 25, 2018 / Published online: September 19, 2018

(C) The Author(s) 2018

\section{ABSTRACT}

Introduction: There is an urgent need to identify patients with bladder cancer (BC) who are at high risk of recurrence or progression. Calgranulin $\mathrm{A}$ is a strong marker for muscle-invasive or advanced $\mathrm{BC}$ and recent studies have shown its potential for identifying patients at

Enhanced digital content To view enhanced digital content for this article go to https://doi.org/10.6084/ m9.figshare.7047752.

\footnotetext{
A. P. Nicklas $(\square)$

Department of Urology and Andrology, Tirol

Kliniken Landeskrankenhaus Hall, Hall in Tirol,

Austria

e-mail: andre.nicklas@tirol-kliniken.at

A. P. Nicklas · J. Serth · M. A. Kuczyk ·

C.-A. J. von Klot

Department of Urology and Urologic Oncology,

Hannover University Medical School, Hannover,

Germany

M. W. Kramer · M. C. Hupe · A. S. Merseburger

Department of Urology, University Hospital

Schleswig-Holstein, Campus Lübeck, Lübeck,

Germany

J. Hennenlotter · A. Stenzl

Department of Urology, Eberhard-Karls-University,

Tübingen, Germany

D. U. Reimer

Department of Obstetrics and Gynecology, Medical

University Innsbruck, Innsbruck, Austria
}

risk even in non-muscle-invasive bladder cancer (NMIBC). The present study examines risks of recurrence and progression dependent on immunostaining with calgranulin A in NMIBC. Methods: Calgranulin A protein expression was evaluated through the immunohistochemistry of 158 randomly selected, transurethrally resected BC specimens of separate patients (pTa 89, pT1 69) using tissue microarrays. KaplanMeier survival analysis and Cox regression were performed to determine whether calgranulin A expression is associated with recurrence-free survival (RFS), progression-free survival (PFS), or cancer-specific survival (CSS).

Results: Calgranulin A expression is significantly different between pTa and pT1 tumors ( $p=0.000$, Mann-Whitney $U$ test) and between tumor grades ( $p=0.015$, Kruskal-Wallis test). Kaplan-Meier estimates produced significant results for low and high calgranulin A expression concerning RFS [5y-RFS $70.4 \pm 4.0 \%$ vs. $35.9 \pm 12.5 \%$, median RFS not reached (NR) vs. $12.0 \pm 4.4$ month, $p=0.029$, log-rank test], PFS (5y-PFS $90.3 \pm 2.7 \%$ vs. $51.5 \pm 14.0 \%$, median PFS NR in both groups, $p=0.000$, log-rank test), and CSS (5y-CSS $92.9 \pm 2.6 \%$ vs. $70.7 \pm 12.4 \%$, median CSS NR in both groups, $p=0.005$, logrank test). Calgranulin A remained an independent factor for RFS ( $p=0.024$, HR 2.43) and PFS ( $p=0.002$, HR 5.92) according to the multivariate Cox regression model. 
Conclusions: Calgranulin A expression in NMIBC, detected through immunohistochemistry, is a promising marker for the identification of NMIBC patients at high risk of recurrence and progression.

Keywords: Bladder cancer; Calgranulin A; Cancer-specific survival; Immunohistochemistry; NMIBC; Oncology; Progression; Recurrence; S100A8; Tissue microarray

\section{INTRODUCTION}

Bladder cancer (BC) has the highest lifetime treatment cost per patient of all cancers [1], amounting in the European Union to approximately $€ 4.9$ billion per year [2]. Approximately $75-85 \%$ of patients with BC present with the disease confined to the mucosa (stage pTa, pTis) or to the submucosa (stage pT1). Of these patients approximately 50\% will have at least one tumor recurrence, and periodic cystoscopies of all these patients are mandatory to detect recurrence as early as possible. Long-term cost benefits can be achieved through reduced tumor recurrence and potentially reduced progression rates [1]. In addition to recurrence, $11 \%$ of initial non-muscle-invasive bladder cancers (NMIBC) will progress to a muscle-invasive stage [3]. High-grade pT1 (formerly pT1 G3) BC in particular has a high propensity to recur and will progress to a muscle-invasive stage in approximately $50 \%$ of patients [4]. The unpredictable biological behavior of NMIBC, including the development of locally invasive tumor growth, the latter mainly a risk for patients with pT1 tumors, is one of the dilemmas faced during the treatment of the disease. Currently, the search for reliable serum- and urine-based, histological or staging criteria that can adequately predict recurring or progressive tumors is ongoing. Identification of patients at high risk is needed to select them for a more aggressive approach to therapy and follow-up.

There are many strategies to classify the risk of recurrence and/or progression of BC. Generally, NMIBC can be stratified into high-, intermediate, and low-risk groups depending on tumor stage, grade, size, number, and recurrence pattern $[3,5]$. Although the strength of the European Organization for Research and Treatment of Cancer (EORTC) risk groups is their excellent database, in individual cases it is sometimes difficult to decide on the basis of the primary tumor occurrence because of the need for the time-dependent factor "recurrence" and because of the likelihood to have understaging in transurethral resection of bladder tumors (TURBT) [6]. Technical improvements in the surgical treatment of NMIBC, such as photodynamic diagnosis-assisted transurethral resection of bladder tumor, are targeted at a reduction in recurrence rates [7]. Many novel tumor markers have been identified and are still being evaluated to improve the accuracy of prognosis and therapy $[8,9]$.

Calgranulin A (S100A8) belongs to the S100 protein family, which has more than 20 members, all carrying a $\mathrm{Ca}^{2+}$-binding EF-Hand motif. Calgranulin A is a protein of $10.83 \mathrm{kD}$ and its gene is located at chromosome 1q21 [10]. It forms a heterodimer with calgranulin $B$ (S100A9) which is called calprotectin. Calgranulin A may function in the inhibition of casein kinase and as a cytokine, and S100 proteins are involved in cell differentiation and cell cycle regulation $[11,12]$. The precise function of the S100 proteins in tumorigenesis and tumor progression is still unknown but recent reports emphasize their role as a regulator of tumor progression [13]. Calgranulin A has been identified as highly expressed in muscle-invasive and advanced BC; however, studies of calgranulin A expression and its role in NMIBC are sparse.

The aim of this study was to assess the risks of recurrence and progression dependent on calgranulin A immunostaining in transurethrally resected bladder cancer specimens for the identification of NMIBC at high risk of recurrence or progression.

\section{METHODS}

This study was performed in consideration of the Declaration of Helsinki [14]. Patient data and specimens were used with informed 
consent, and this was approved by the ethics committee of the medical faculty at the University of Tübingen, Germany.

\section{Patients}

This study included a total of 158 archival histological specimens of separate patients with primary NMIBC (pTa and pT1), treated by transurethral resection between 1993 and 2002. In this study, specimen selection was based solely on the availability of well-preserved tumor tissue blocks and the availability of enough tissue in those blocks for microarray preparation. A database of patients with paraffin blocks of the resected bladder tumor available was first created. Only patients with a minimum follow-up of 3 years following initial transurethral treatment of the lesion or in case of tumor recurrence or progression regardless of the time when either recurrence or progression occurred were included. All tumors were initially diagnosed by pathologists and graded according to the 1973 WHO grading system [15]. As the sample collection was all performed before the implementation of the 2004 WHO grading system for urothelial cancers, the new classification could not be used in the present study. Patient data and follow-up information were collected retrospectively by searching the clinical information system. Missing follow-up data was collected by written inquiry to the referring urologists. Inpatient treatment and outpatient follow-up were performed according to the EAU guidelines on non-muscle-invasive urothelial carcinoma of the bladder [16]. Follow-up conforming to the guidelines was recommended to all referring urologists, who performed followups themselves, but data about the reliability of the recommended follow-up is lacking. The same cohort was validated in four previous biomarker studies investigating galectin-3 [17], HYAL-1 hyaluronidase [18], maspin [19], and galectin-8 [20]. Recurrence was defined as the reappearance of a tumor at the same tumor stage. Recurrence-free follow-up was assumed if there was no tumor reported in routine cystoscopies, performed either at the hospital or by the referring urologist. Progression was defined as tumor development to a higher tumor stage, and cancer-specific death was presumed if the patient died within the follow-up period as a result of progressive bladder cancer disease. In the 158 cases studied, clinicopathologic parameters and a complete follow-up were available. The median follow-up interval was 49.5 months and the last follow-up data was collected in 2009.

Twenty-four tumor specimens from patients with muscle-invasive bladder cancer $(\geq$ pT2) were also analyzed to investigate differential staining patterns, in contrast to non-muscleinvasive tumor specimens. In these cases, clinical data was available, but follow-up data was not suitable for inclusion in our assessment of recurrence and progression, because of the divergent therapies these patients underwent. These 24 patients with muscle-invasive tumors were therefore not included in the survival analyses, but they were additionally examined in the assessment of calgranulin staining levels regarding tumor stage and grade.

\section{Tissue Microarray and Immunohistochemical Analysis}

The 158 non-muscle-invasive and 24 muscleinvasive tumor specimens were routinely formalin-fixed, dehydrated, and embedded in paraffin. Hematoxylin and eosin staining was performed on $4-\mu \mathrm{m}$ sections for each tumor specimen to validate tumor stage and grade, as well as for the onset of tissue microarrays (TMA). The TMAs were constructed as described previously $[21,22]$. TMA slides were stained for S100A8 protein expression using a monoclonal antibody to calgranulin A (Mouse/IgG2b Clone: CF-145, Acris Antibodies GmbH, Herford, Germany). Sections were demasked by incubation at $60^{\circ} \mathrm{C}$ using a $10 \mathrm{mM}$ citrate buffer ( $\mathrm{pH} \mathrm{6.0)}$. After the blocking of endogenous peroxidases, the sections were treated using the avidin/biotin blocking kit (Vector Laboratories, Burlingame, CA, USA) to block endogenous biotin as well as unspecific avidin binding. Following treatment in $4 \%(\mathrm{w} / \mathrm{v})$ nonfat milk for $30 \mathrm{~min}$, the slides were incubated with the monoclonal antibody to calgranulin A (dilution 1:60, 
incubation overnight at room temperature). The sections were stained using the standard avidin-biotin system according to the manufacturer's protocols. Signal amplification using tyramide was carried out as described previously $[23,24]$. The tissue sections were counterstained with hematoxylin. To rule out false positive results, a single tissue microarray slide played the role of another negative control and was immunohistochemically processed as described above, except for the antibody incubation step. No signals were obtained in this additional negative control slide. Positive control was performed by staining well-defined BC samples of muscle-invasive tumors, approved and staged by a pathologist from our institution. All positive control samples showed full and intensive staining of the tumor. TMA sections were reviewed and rated after immunohistochemical staining by two independent investigators (APN, MWK), completely blinded to the clinical data and follow-up information. A regular bright-field microscope was used to determine the grading index of the TMA staining. We used a system of TMA grading indexing between 0 and 300, as described previously [21, 25]. All cases where results differed between the two investigators were double-checked, and the calgranulin A staining levels were finally determined by consensus, still blinded to the clinical data.

\section{Statistical Analysis}

Statistical analysis was performed using IBM $^{\circledR}$ SPSS $^{\circledR}$ Statistics Version 23 (IBM Corporation, Armonk, NY, USA) and the open-source statistical software "R", version 3.4.3 (https://www.Rproject.org). Continuous variables are expressed as mean and standard deviation, or median and range, and categorical variables are expressed with absolute and relative frequencies. Mean and median values were reported after visual assessment of data via histogram plots, with normally distributed values reported with means and standard deviation and non-normally distributed values reported with medians and range. Time-dependent variables as well as patient age were reported with medians and range. Additionally, the Kolmogorov-Smirnov test was used to evaluate distributions of calgranulin A staining levels. At each test the null hypothesis was assuming that there is no difference between the test variables, and $p$ values less than 0.05 were considered statistically significant resulting in rejection of the null hypothesis. The Mann-Whitney $U$ test and Kruskal-Wallis tests were performed to evaluate calgranulin A staining levels according to varying clinical variables. Correlation between age and calgranulin A staining index was assessed with Spearman's rank correlation coefficient. The cutoff determination was explicitly performed after data acquisition and prior to statistical first statistical analysis. The "Significance of correlation with survival variable" method in the cutoff finder tool from Budczies et al. [26] was used for cutoff determination: this method fits Cox proportional hazard models for the dichotomized variable and the survival variable. Hazard ratios (HRs) were calculated with 95\% confidence intervals (CI). Prognostic evaluation was performed using Kaplan-Meier survival analysis and log-rank tests. Cox proportional hazards regression was used to assess the independent prognostic value of calgranulin A expression levels.

\section{RESULTS}

\section{Assessment of Immunohistochemical Staining of Calgranulin A}

Only the staining reaction within tumor cells was used for the classification of the immunohistochemical staining patterns. Calgranulin A expression was preferably detected within the cytoplasm of tumor cells (Fig. 1). In a few cases, nuclear staining was observed, however, this revealed no prognostic information for the present cohort of patients.

\section{Correlation of Calgranulin A Expression and Clinicopathological Parameters}

Of the 158 NMIBC specimens included in the present investigation, $122(77.2 \%)$ were from 


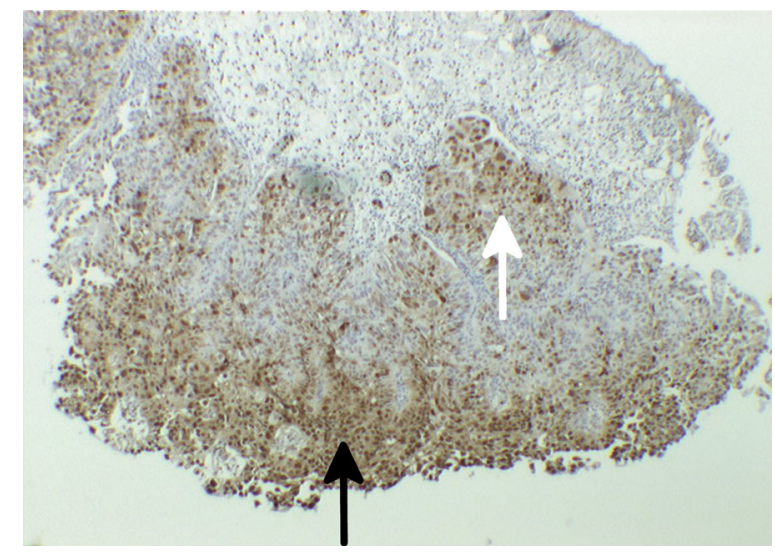

Fig. 1 Calgranulin A expression detected immunohistochemically using anti-S100A8 mAb. Arrows indicate positive staining of epithelial (black) and subepithelial (white) tumor cells

male and 36 (22.8\%) from female patients. The median age at diagnosis was 69.0 years (range 32-90) and the median follow-up interval was 49.5 months (range 4-139). According to histopathologic evaluation, tumor stages were classified as pTa in $89(56.3 \%)$ and pT1 in 69 (43.7\%). Concomitant carcinoma in situ (pTis) was found in 7 (4.4\%) patients, one in stage pTa and six in stage pT1. The tumor grades of the cases included in the prognostic evaluation were as follows: G1 in 60 (38.0\%) cases, G2 in 86 (54.4\%), and G3 in 12 (7.6\%) (Table 1). Twentyfour cases with stage $\geq \mathrm{pT} 2$ were compared to the NMIBC specimens. Consequently, 182 specimens were included in the analysis of calgranulin A expression regarding tumor stage and grade, of which $89(48.9 \%)$ were pTa, 69 $(37.9 \%)$ pT1, and $24(13.2 \%) \geq \mathrm{pT} 2$. The distribution of tumor grades in the 182 cases was G1 in $60(33.0 \%)$, G2 in $93(51.1 \%)$, and G3 in 29 (15.9\%) cases.

Calgranulin A expression patterns were tested for various patient and tumor characteristics, such as patient's age and gender, the detection of pTis in addition to the primary papillary lesion, multifocality, and tumor stage and grade. There was no positive or negative correlation between patient age and calgranulin A expression levels (sample size 158, Spearman's correlation coefficient $r_{\mathrm{s}}=0.13, p=0.103$ ). Moreover, there were no significant differences in calgranulin A expression levels for gender (sample size 158, $p=0.108$, Mann-Whitney $U$ test). Furthermore, patients with or without multifocal tumor growth showed no significant differences in calgranulin A expression levels (sample size 158, $p=0.075$, Mann-Whitney $U$ test). Because the results for age, gender, and multifocality were close to significance, further tests of these variables were also included in the Kaplan-Meier survival analysis. Simultaneous detection of pTis in the specimens showed

Table 1 Distribution of clinicopathological parameters

\begin{tabular}{llll}
\hline Parameter & $N$ & $\%$ & $\begin{array}{l}\text { Mean calgranulin } \\
\text { A expression } \\
\end{array}$ \\
& & level $(0-300)$ \\
\hline
\end{tabular}

Tumor stage

$\begin{array}{lrrr}\mathrm{Ta}+\mathrm{T} 1 & 158 & 100.0 & 26.13 \\ \mathrm{Ta} & 89 & 56.3 & 8.26 \\ \mathrm{~T} 1 & 69 & 43.7 & 49.19\end{array}$

Concomitant $\mathrm{pT}$ is

$\begin{array}{lrrr}\text { No } & 151 & 95.6 & 24.83 \\ \text { Yes } & 7 & 4.4 & 54.29\end{array}$

Tumor size $(\mathrm{cm})$

$\begin{array}{rrrr}<3 & 129 & 81.6 & 28.53 \\ \geq 3 & 8 & 5.1 & 0.83\end{array}$

Multifocality

$\begin{array}{rrrr}\text { No } & 104 & 65.8 & 19.57 \\ \text { Yes } & 54 & 34.2 & 38.77\end{array}$

Tumor grade

$\begin{array}{rrrr}\text { G1 } & 60 & 38.0 & 13.93 \\ \text { G2 } & 86 & 54.4 & 33.47 \\ \text { G3 } & 12 & 7.6 & 34.58\end{array}$

Gender

\begin{tabular}{lrrr} 
Male & 122 & 77.2 & 20.17 \\
Female & 36 & 22.8 & 46.34 \\
Age (years) & & & \\
$\leq 69.0$ & 80 & 50.6 & 18.82 \\
$>69.0$ & 78 & 49.4 & 33.63 \\
\hline
\end{tabular}


significant differences in calgranulin A expression levels when compared to specimens without pTis (sample size 158, $p=0.030$, Mann-Whitney $U$ test).

Higher calgranulin A expression was significantly associated with a higher tumor stage (sample size 182, $p=0.000$, Kruskal-Wallis test) and shows significant difference between pTa and pT1 lesions (sample size 158, $p=0.000$, Mann-Whitney $U$ test). Calgranulin A expression was also significantly divergent between tumor grades (sample size 182, $p=0.015$, Kruskal-Wallis test) (Fig. 2).
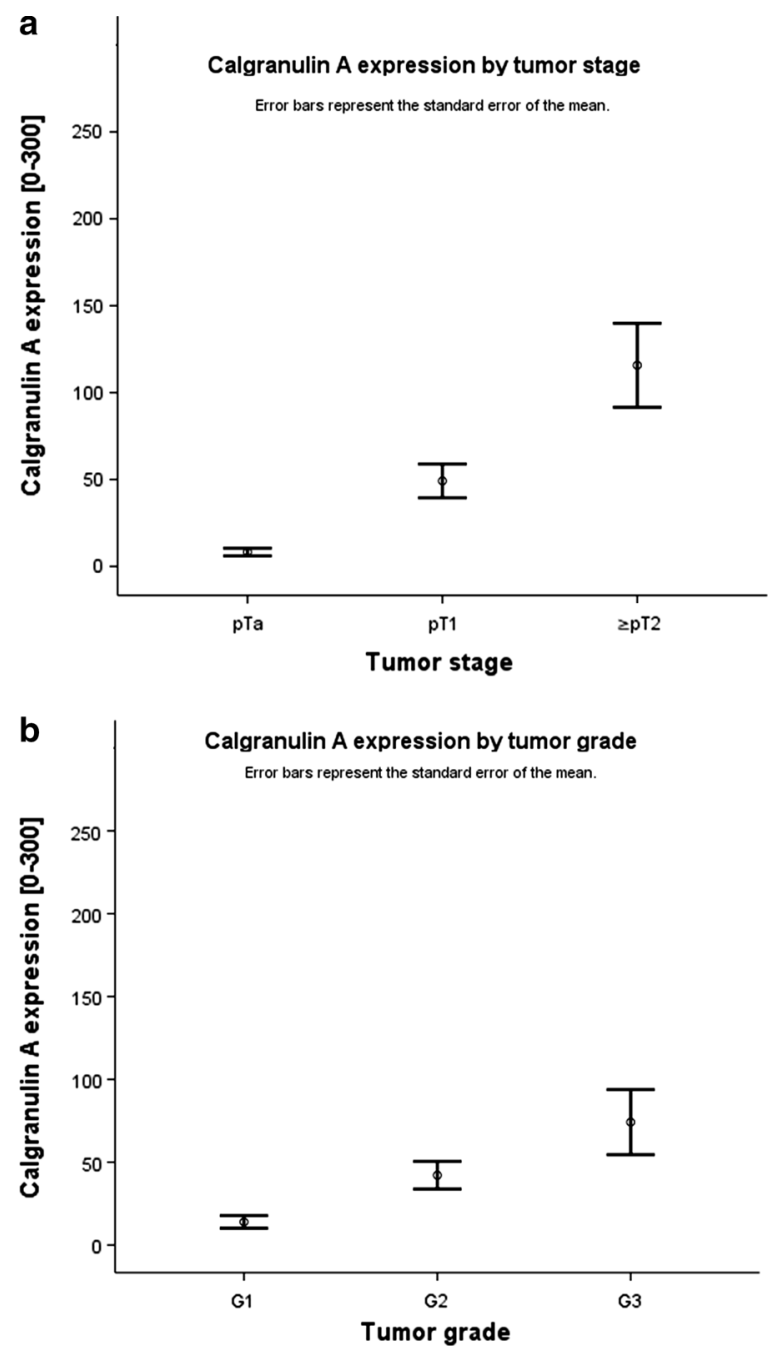

Fig. 2 Calgranulin A expression was significantly associated with a higher tumor stage (a $p=0.000$; Kruskal-Wallis test) and tumor grade (b $p=0.015$; Kruskal-Wallis test)

\section{Correlation of Calgranulin A Expression and Follow-up Data (Recurrence-Free Survival, Progression-Free Survival, Cancer-Specific Survival)}

Information about RFS, PFS, and CSS at the end of the follow-up period was available in 158, 158 , and 145 cases, respectively. Recurrence occurred in $67(42.4 \%)$ patients, of which 39 $(58.2 \%)$ had stage pTa and $28(41.8 \%)$ stage pT1. Progression developed in 24 (15.2\%) of the patients. In the group with progression, 11 $(45.8 \%)$ had stage pTa and $13(54.2 \%)$ stage pT1. The median time to recurrence was 10 months (range 3-72) and the median time to progression was 11 months (range 0-79). Cancerspecific death occurred in 14 (8.9\%) patients and the median time to cancer-specific death was 29.0 months (range 6-83).

Cutoff calculation resulted in different cutoff values of calgranulin A staining index: 95 for RFS and 108 for PFS and CSS. Calgranulin A staining indices less than the cutoff values were classified as low calgranulin A expression, and values greater than or equal to the cutoff as high calgranulin A expression.

In the Kaplan-Meier survival analysis we found significant differences between low and high calgranulin A expression in terms of RFS (sample size 158, 5y-RFS $70.4 \pm 4.0 \%$ vs. $35.9 \pm 12.5 \%$, median RFS not reached (NR) vs. $12.0 \pm 4.4$ month (95\% CI 3.3-20.7), $p=0.029$, log-rank test), PFS (sample size 158, 5y-PFS $90.3 \pm 2.7 \%$ vs. $51.5 \pm 14.0 \%$, median PFS NR in both groups, $p=0.000$, log-rank test), and CSS (sample size 145, 5y-CSS $92.9 \pm 2.6 \%$ vs. $70.7 \pm 12.4 \%$, median CSS NR in both groups, $p=0.005$, log-rank test) (Fig. 3). Moreover, Kaplan-Meier survival analysis for RFS dependent on age ( $<69$ years vs. $\geq 69$ years) showed no significant difference (sample size 158, 5yRFS $59.5 \pm 5.9 \%$ vs. $50.0 \pm 6.1 \%$, median RFS NR vs. $40.0 \pm 13.0$ month (95\% CI 14.4-65.6), $p=0.103$, log-rank test). On the other hand, Kaplan-Meier survival analysis for PFS (sample size 158 , 5 -RFS $88.2 \pm 3.9 \%$ vs. $50.0 \pm 6.1 \%$, median RFS NR in both groups, $p=0.030$, logrank test) and CSS (sample size 145, 5y-RFS $91.9 \pm 4.2 \%$ vs. $76.4 \pm 7.2 \%$, median RFS NR in both groups, $p=0.036$, log-rank test) resulted in 
significant differences dependent on age. Furthermore, there were no significant differences in Kaplan-Meier survival analysis comparing gender (male vs. female) against RFS (sample size $158,5 y$-RFS $49.7 \pm 5.0 \%$ vs. $62.8 \pm 8.9 \%$, median RFS NR in both groups, $p=0.423$, logrank test), PFS (sample size 158, 5y-RFS $83.6 \pm 3.9 \%$ vs. $75.1 \pm 8.6 \%$, median RFS NR in both groups, $p=0.357$, log-rank test), and CSS (sample size: $145, \quad 5 y-R F S \quad 91.0 \pm 3.4 \%$ vs. $81.8 \pm 7.5 \%$, median RFS NR in both groups, $p=0.233$, log-rank test). Surprisingly, KaplanMeier survival analysis comparing unifocal tumors vs. multifocal tumors were not significant for RFS (sample size 158, 5y-RFS $57.7 \pm 5.4 \%$ vs. $44.1 \pm 7.2 \%$, median RFS not reached $(\mathrm{NR})$ vs. $26.0 \pm 15.6$ month $(95 \% \mathrm{CI}$ 0.0-56.6), $p=0.151$, log-rank test) and CSS (sample size $145,5 y-R F S \quad 88.4 \pm 4.0 \%$ vs. $89.9 \pm 4.8 \%$, median RFS not NR in both groups, $p=0.892$, log-rank test), but resulted in a significant difference concerning PFS (sample size $158,5 y$-RFS $86.2 \pm 4.0 \%$ vs. $74.1 \pm 6.7 \%$, median RFS not NR in both groups, $p=0.044$, log-rank test).

Cox proportional hazard analysis was performed to evaluate independent variables for RFS, PFS, and CSS (Table 2). As the limited number of events concerning recurrence $(n=67)$, progression $(n=24)$, and cancerspecific deaths $(n=14)$ in our dataset was a delimiter for the number of covariates in multivariate Cox regression, we decided to determine the covariates based on the already existing EORTC risk factors (without prior recurrence rate, because of the fact our cases contained only primary tumors). Covariates in the Cox proportional hazard analysis were calgranulin A expression (low vs. high), tumor stage (pTa vs. pT1), concomitant pTis (no vs. yes), tumor size $(<3 \mathrm{~cm}$ vs. $\geq 3 \mathrm{~cm})$, number of tumors, and tumor grade (G1 vs. G2 or G3). In the univariate Cox regression calgranulin $\mathrm{A}$ expression was consistently the only significant factor among the chosen covariates regarding RFS (sample size 158, $p=0.036$, HR 2.06), PFS (sample size 158, $p=0.000$, HR 5.35), and CSS (sample size $145, p=0.011, \mathrm{HR} 4.55$ ). In the multivariate Cox regression calgranulin A expression remained an independent factor for
RFS (sample size 158, $p=0.024$, HR 2.43) and PFS (sample size 158, $p=0.002$, HR 5.92), but failed to be significant in CSS (sample size 145, $p=0.147$, HR 3.24).

\section{DISCUSSION}

The main predicament of NMIBC remains the high percentage of patients who will suffer recurrence or progression. Patients with pTa tumors have a likelihood of recurrence from $50 \%$ to $80 \%$, but on the other hand, the main threat of the pT1 and pTis stages is the risk of progression to a higher stage (10-30\%) [27]. Some patients with pT1G3 BC will undergo lifelong invasive surveillance without any recurrence after therapy [28]. It is crucial for the outcome of the disease to identify those tumors with high risk of recurrence and progression, in order to adjust the treatment or surveillance strategy according to individual risk. The data most commonly used to predict the further outcome and treatment of $\mathrm{BC}$ is currently clinicopathological, such as the tumor stage, tumor grade, size of the tumor, multifocality, and prior recurrence rate. To quantify the risk and implement risk groups, EORTC developed a scoring system based on six variables to calculate the probability of recurrence (score from 0 to 17) and progression (score from 0 to 23), using data from 2596 patients who participated in seven EORTC trials [3]. On the basis of these scores, the European Association of Urology (EAU) defined risk groups for recurrence and progression in their guidelines [29]. Different prognostic models and nomograms for risk grouping, using clinicopathological patterns, have been published for NMIBC and have been widely used in clinical practice, starting in 1989 with the British Medical Research Council Subgroup on Superficial Bladder Cancer [30], later with the Spanish group led by Millan-Rodriguez [5], and recently by Sylvester's EORTC risk tables [3]. In contrast to the use of clinicopathological parameters alone, the nomogram of Shariat et al., which uses age, gender, urine cytology, and a urine-based biomarker test (nuclear matrix protein, NMP22), can predict the probability of recurrence and progression in 

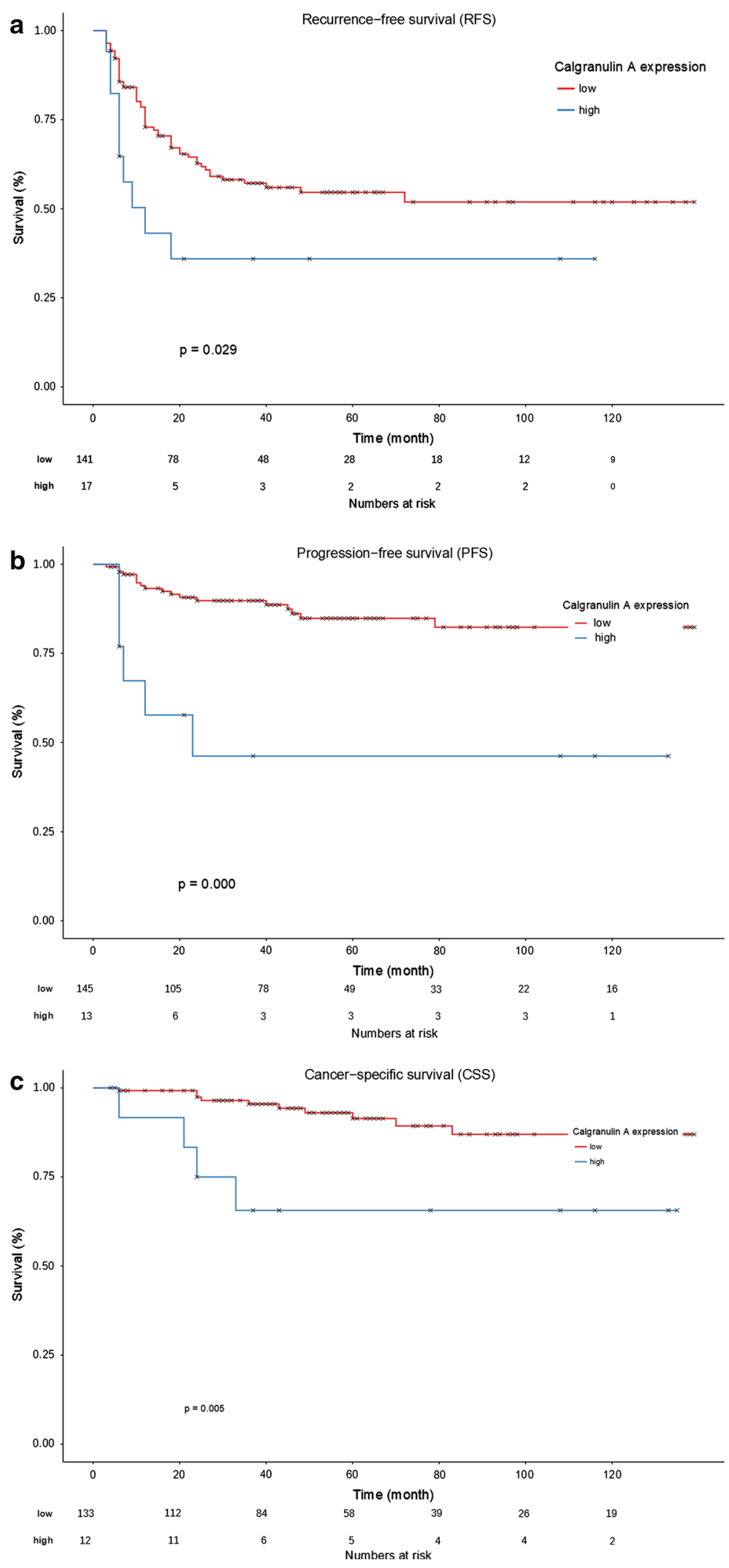
4 Fig. 3 Kaplan-Meier survival analysis shows significant differences between low and high calgranulin A expression in terms of RFS (a $p=0.029$, log-rank test), PFS (b $p=0.000, \log$-rank test), and CSS (c $p=0.005, \log -$ rank test)

NMIBC with high accuracy [31]. Recent findings have demonstrated that NMIBC can be grouped into three major subclasses with basal- and luminal-like characteristics and different clinical outcomes [32]. Finally, a recent report highlighted the assessment and reporting of lymphovascular invasion and variant histology in TUR specimens as important for risk stratification and decision-making [33].

In search of novel prognostic tools, a great variety of biomarkers have been examined. For implementation in routine clinical practice, a prognostic marker should be simple and costeffective, such as the immunostaining of pathological specimens.

Calgranulin A has been shown to be associated with invasive and metastatic tumor growth. The association of the deregulation of S100A8 has also been demonstrated in prostate (sample size 75; immunohistochemistry) [34], colon (sample size 23 matched pairs; 2D-PAGE and MALDI-MS) [35], gastric (sample size 218; TMA immunohistochemistry) [36], and gall bladder cancer (sample size NA; two-dimensional liquid chromatography and tandem mass spectrometry) [37].

Tolson et al. used the SELDI-TOF analysis of 12 pairwise $\mathrm{BC}$ specimens, comparing muscleinvasive $\mathrm{BC}$ biopsies with homologous normal tissue, to demonstrate that calgranulin $\mathrm{A}$ is highly overexpressed within tumor cells, and suggested a prognostic value of S100A8 in BC [38]. A systematic evaluation of the $\mathrm{S} 100$ protein family, using microarray and real-time PCR in murine and human $\mathrm{BC}$ specimens (sample size NA), detected S100A8 overexpression in comparison to normal bladder tissue [39]. Associations with stage progression, invasion, metastasis, and poor survival have also been presented in a review by Yao et al. [13]. Pilchowski et al. identified calgranulin $\mathrm{A}$ as significantly overexpressed in metastatic BC compared to non-metastatic muscle-invasive BC specimens, via Protein Chip technology surface enhanced laser desorption/ionization time of flight mass spectrometry (sample size 88) [40]. In a proteomic study from Minami et al. comparing the pre- and postoperative sera and bladder tumor specimens from $77 \mathrm{BC}$ patients, S100A8 was identified as associated with muscle-layer tumor invasion and cancerspecific survival [41]. Ebbing et al. detected urinary calprotectin concentrations significantly higher in patients with bladder cancer than in healthy controls (sample size 181) [42]. Finally, a microarray gene expression profiling study by Kim et al., using a four-gene signature including S100A8 gene expression levels, showed that these gene signatures could significantly predict the progression of muscle-invasive bladder tumors (sample size 128) [43].

There is still sparse information for NMIBC about the usefulness of calgranulin $\mathrm{A}$ as a prognostic tool. Ha et al. offered the first evidence for this, examining the mRNA expression of S100A8 in 103 NMIBC tissue samples, and concluding that calgranulin A might be a useful prognostic marker for progression to MIBC [44]. Kim et al. illustrated that the microarray gene expression profiling of 103 tissue samples is a promising diagnostic tool for the identification of NMIBC patients with a high risk of progression to MIBC [45]. Bansal et al. detected significant differences in calgranulin A expression between low-grade and high-grade BC in 160 preoperatively examined patients, using serumbased proteomics [46], and could even show that expression levels were gradually and significantly reduced after surgical treatment [47].

In the present study, the immunostaining of tumor cells with an antibody against calgranulin A showed significant differences regarding the tumor stage and grade of patient specimens. It therefore appears that its expression positively correlates with tumor invasiveness and differentiation, as demonstrated by previous research groups [38-40]. Remarkably, calgranulin A expression levels in NMIBC, observed via immunostaining, are very low, with $66.5 \%$ of all superficial tumor specimens showing no calgranulin A expression at all (calgranulin A staining index $=0$ ). The overexpression of 
Table 2 Cox proportional hazard models

\begin{tabular}{|c|c|c|c|}
\hline Parameter & Hazard ratio & $95 \%$ confidence interval & $p$ value \\
\hline \multicolumn{4}{|l|}{ Recurrence-free survival (RFS) } \\
\hline \multicolumn{4}{|l|}{ Univariate Cox regression } \\
\hline Calgranulin A expression & 2.06 & $1.05-4.04$ & 0.036 \\
\hline Tumor stage & 1.06 & $0.65-1.72$ & 0.815 \\
\hline Concomitant $\mathrm{p}$ Tis & 0.63 & $0.15-2.55$ & 0.513 \\
\hline Tumor size & 0.58 & $0.14-2.40$ & 0.455 \\
\hline Multifocality & 1.11 & $0.86-1.45$ & 0.426 \\
\hline Tumor grade & 1.03 & $0.63-1.67$ & 0.916 \\
\hline \multicolumn{4}{|l|}{ Multivariate Cox regression } \\
\hline Calgranulin A expression & 2.43 & $1.12-5.25$ & 0.024 \\
\hline Tumor stage & 0.95 & $0.48-1.89$ & 0.883 \\
\hline Concomitant $\mathrm{pT}$ is & 1.05 & $0.24-4.51$ & 0.949 \\
\hline Tumor size & 0.69 & $0.16-2.98$ & 0.615 \\
\hline Multifocality & 1.18 & $0.88-1.58$ & 0.262 \\
\hline Tumor grade & 0.98 & $0.52-1.83$ & 0.948 \\
\hline \multicolumn{4}{|l|}{ Progression-free survival (PFS) } \\
\hline \multicolumn{4}{|l|}{ Univariate Cox regression } \\
\hline Calgranulin A expression & 5.35 & $2.11-13.57$ & 0.000 \\
\hline Tumor stage & 1.80 & $0.80-4.04$ & 0.156 \\
\hline Concomitant $\mathrm{p}$ Tis & 1.11 & $0.15-8.24$ & 0.919 \\
\hline Tumor size & 0.05 & $0.00-118.45$ & 0.441 \\
\hline Multifocality & 1.24 & $0.08-1.86$ & 0.291 \\
\hline Tumor grade & 0.75 & $0.33-1.67$ & 0.476 \\
\hline \multicolumn{4}{|l|}{ Multivariate Cox regression } \\
\hline Calgranulin A expression & 5.92 & $1.94-18.01$ & 0.002 \\
\hline Tumor stage & 1.68 & $0.55-5.15$ & 0.366 \\
\hline Concomitant $\mathrm{pTis}$ & 2.07 & $0.26-16.63$ & 0.493 \\
\hline Tumor size & 0.00 & 0.00 & 0.980 \\
\hline Multifocality & 1.15 & $0.74-1.78$ & 0.532 \\
\hline Tumor grade & 0.38 & $0.13-1.15$ & 0.088 \\
\hline
\end{tabular}

Cancer-specific survival (CSS)

Univariate Cox regression

Calgranulin A expression

4.55

$1.42-14.53$

0.011 
Table 2 continued

\begin{tabular}{llll}
\hline Parameter & Hazard ratio & $\mathbf{9 5 \%}$ confidence interval & $\boldsymbol{p}$ value \\
\hline Tumor stage & 2.13 & $0.73-6.18$ & 0.166 \\
Concomitant pTis & 1.84 & $0.23-14.68$ & 0.567 \\
Tumor size & 0.05 & $0.00-6481.82$ & 0.610 \\
Multifocality & 0.77 & $0.36-1.67$ & 0.505 \\
Tumor grade & 2.29 & $0.71-7.35$ & 0.166 \\
Multivariate Cox regression & & & 0.147 \\
Calgranulin A expression & 3.24 & $0.66-15.81$ & 0.774 \\
Tumor stage & 1.25 & $0.27-5.83$ & 0.992 \\
Concomitant pTis & 0.00 & 0.00 & 0.990 \\
Tumor size & 0.00 & 0.00 & 0.462 \\
Multifocality & 0.72 & $0.29-1.75$ & 0.593 \\
Tumor grade & 1.47 & $0.34-6.00$ & \\
\hline
\end{tabular}

Cox proportional hazard models for calgranulin A expression and various histological parameters: high calgranulin A expression is significantly associated with RFS $(p=0.036)$, PFS $(p=0.000)$, and CSS $(p=0.011)$. In multivariate Cox regression calgranulin A expression is an independent prognostic factor for RFS $(p=0.024)$ and PFS $(p=0.002)$

calgranulin A in NMIBC emerged as a potential prognostic factor, however, and Kaplan-Meier estimates showed significant results for recurrence, progression, and CSS. This suggests a prognostic value, as Cox multivariate regression highlighted calgranulin A expression as an independent prognostic factor for recurrence and progression.

This investigation was a preliminary test to translate the increasingly identified usefulness of calgranulin A as a prognostic marker in BC into clinical routine. Immunostaining with calgranulin A could be performed in routine histopathological assessment, to identify patients with NMIBC and a high risk for recurrence and progression.

This study is the first presentation of calgranulin A immunostaining in NMIBC and is a preliminary test because of its limitations: the small number of patients cannot compete with prognostic considerations as derived for instance from the current EORTC risk tables that comprise data from over 2500 patients [3]. However, calgranulin A showed significant time/event differences with our smaller dataset already, hence follow-up research on this marker should be interesting. Another shortcoming of the work is its retrospective design and the need to calculate the cutoff value retrospectively. Additionally, the quality of our follow-up data may be influenced by the lack of a standardized follow-up protocol: follow-up according to the EAU guidelines was requested, but we could not monitor the reliability of guideline conformity in some cases, when referring urologists did the follow-up themselves. Since the 2004 (and 2010) WHO grading system for urothelial cancers has brought distinct changes compared to the 1973 WHO grading system, which still was used in the study, it will be difficult to compare our data with recent cohorts because of overlapping of G2 grading and low- and high-grade tumor grading, respectively. In this regard, keeping the 1973 WHO grading in pathological reports together with the new grading is important. Since age, gender, and multifocality have been proposed as prognostic factors in the past, we assume that different results than ours, e.g., that age, gender, and multifocality were not 
constantly significant in Kaplan-Meier survival analysis, may arise with higher patient numbers in a possible validation study. Restricting the Cox proportional hazard analysis to the factors of the EORTC risk tables may influence the results of the multivariate regression, because other factors, e.g., age and gender, have already been identified as prognostically relevant. Because the number of events in our dataset was a limiting factor, this has to be validated in larger cohorts.

Further studies are needed to define the reliability and prognostic value of immunostaining with calgranulin A in NMIBC. These should be based on a larger cohort of patients, at best multicentric, and should be designed with a prospective setting. A comparison of calgranulin A expression, detected through immunostaining, with expression levels assessed by other techniques, e.g., mRNA expression or serum-based proteomics, would consolidate the findings. Before clinical acceptance of calgranulin A as a prognostic marker in NMIBC, a defined follow-up schedule should be executed in a prospective setting to make data suitable for calculating positive and negative predictive values in a time-dependent predictive model.

\section{CONCLUSIONS}

Calgranulin A expression in NMIBC, detected by immunohistochemistry, is a promising marker for the identification of NMIBC patients at high risk of recurrence and progression.

\section{ACKNOWLEDGEMENTS}

Funding. No funding or sponsorship was received for this study or publication of this article.

Medical Writing, Editorial, and Other Assistance. Editorial support (native English proof reading) was provided by Proof-ReadingService.com, Devonshire Business Centre, Works Road, Letchworth Garden City, SG6 1GJ,
Hertfordshire, United Kingdom funded by the first author (APN).

Authorship. All named authors meet the International Committee of Medical Journal Editors (ICMJE) criteria for authorship for this article, take responsibility for the integrity of the work as a whole, and have given their approval for this version to be published.

Authorship Contributions. Conception and design: APN, AS, ASM, MAK; C-AJvK; Acquisition of data: APN, MWK, JS, JH; Data analysis and interpretation: APN, MCH, DUR, ASM, C-AJvK. The first draft was written by the lead author, APN. All authors were involved in development of all stages of the manuscript, revising it critically for important intellectual content. All authors provided final approval of the version submitted for publication. All authors are accountable for the accuracy and integrity of the work.

Disclosures. André Philippe Nicklas, Mario Wolfgang Kramer, Jürgen Serth, Jörg Hennenlotter, Marie Christine Hupe, Daniel Uwe Reimer, Arnulf Stenzl, Axel Stewart Merseburger, Markus Antonius Kuczyk, and Christoph-Alexander Joachim von Klot declare that they have nothing to disclose.

Compliance with Ethics Guidelines. All procedures performed in studies involving human participants were in accordance with the ethical standards of the institutional and/or national research committee and with the 1964 Helsinki declaration and its later amendments or comparable ethical standards. Informed consent was obtained from all individual participants included in the study. This article does not contain any studies with animals performed by any of the authors.

Data Availability. The datasets generated during and/or analyzed during the current study are available from the corresponding author on reasonable request.

Open Access. This article is distributed under the terms of the Creative Commons 
Attribution-NonCommercial 4.0 International License (http://creativecommons.org/licenses/ by-nc/4.0/), which permits any noncommercial use, distribution, and reproduction in any medium, provided you give appropriate credit to the original author(s) and the source, provide a link to the Creative Commons license, and indicate if changes were made.

\section{REFERENCES}

1. Sievert KD, Amend B, Nagele U, et al. Economic aspects of bladder cancer: what are the benefits and costs? World J Urol. 2009;27(3):295-300.

2. Leal J, Luengo-Fernandez R, Sullivan R, Witjes JA. Economic burden of bladder cancer across the European Union. Eur Urol. 2016;69(3):438-47.

3. Sylvester RJ, van der Meijden AP, Oosterlinck W, et al. Predicting recurrence and progression in individual patients with stage Ta T1 bladder cancer using EORTC risk tables: a combined analysis of 2596 patients from seven EORTC trials. Eur Urol. 2006;49(3):466-77.

4. Kulkarni GS, Hakenberg OW, Gschwend JE, et al. An updated critical analysis of the treatment strategy for newly diagnosed high-grade T1 (previously T1G3) bladder cancer. Eur Urol. 2009;57(1):60-70.

5. Millan-Rodriguez F, Chechile-Toniolo G, SalvadorBayarri J, Palou J, Algaba F, Vicente-Rodriguez J. Primary superficial bladder cancer risk groups according to progression, mortality and recurrence. J Urol. 2000;164(3Pt1):680-4.

6. Minardi D, Milanese G, Parri G, Lacetera V, Muzzonigro G. Non-muscle invasive high grade urothelial carcinoma of the bladder. Which factors can influence understaging at the time of radical cystectomy? Arch Ital Urol Androl. 2016;88(1):13-6.

7. Gallagher KM, Gray K, Anderson CH, et al. 'Real-life experience': recurrence rate at 3 years with Hexvix ${ }^{\circledR}$ photodynamic diagnosis-assisted TURBT compared with good quality white light TURBT in new NMIBC-a prospective controlled study. World J Urol. 2017;35(12):1871-7.

8. Frantzi M, Latosinska A, Fluhe L, et al. Developing proteomic biomarkers for bladder cancer: towards clinical application. Nat Rev Urol. 2015;12(6):317-30.

9. Latosinska A, Frantzi M, Vlahou A, Merseburger AS, Mischak H. Clinical proteomics for precision medicine: the bladder cancer case. Proteomics Clin Appl. 2018;12(2):1700074.

10. Marenholz I, Heizmann CW, Fritz G. S100 proteins in mouse and man: from evolution to function and pathology (including an update of the nomenclature). Biochem Biophys Res Commun. 2004;322(4):1111-22.

11. O'Leary NA, Wright MW, Brister JR, et al. Reference sequence (RefSeq) database at NCBI: current status, taxonomic expansion, and functional annotation. Nucleic Acids Res. 2016;44(D1):D733-45.

12. Donato R. Intracellular and extracellular roles of S100 proteins. Microsc Res Tech. 2003;60(6):540-51.

13. Yao R, Davidson DD, Lopez-Beltran A, MacLennan GT, Montironi R, Cheng L. The S100 proteins for screening and prognostic grading of bladder cancer. Histol Histopathol. 2007;22(9):1025-32.

14. World Medical Association. World Medical Association Declaration of Helsinki: ethical principles for medical research involving human subjects. JAMA. 2013;310(20):2191-4.

15. Sobin LH. The WHO histological classification of urinary bladder tumours. Urol Res. 1978;6(4):193-5.

16. Babjuk M, Oosterlinck W, Sylvester R, Kaasinen E, Bohle A, Palou-Redorta J. EAU guidelines on nonmuscle-invasive urothelial carcinoma of the bladder. Eur Urol. 2008;54(2):303-14.

17. Kramer MW, Kuczyk MA, Hennenlotter J, et al. Decreased expression of galectin-3 predicts tumour recurrence in pTa bladder cancer. Oncol Rep. 2008;20(6):1403-8.

18. Kramer MW, Golshani R, Merseburger AS, et al. HYAL-1 hyaluronidase: a potential prognostic indicator for progression to muscle invasion and recurrence in bladder cancer. Eur Urol. 2010;57(1):86-93.

19. Kramer MW, Waalkes S, Hennenlotter J, et al. Maspin protein expression correlates with tumor progression in non-muscle invasive bladder cancer. Oncol Lett. 2010;1(4):621-6.

20. Kramer MW, Waalkes S, Serth J, et al. Decreased galectin- 8 is a strong marker for recurrence in urothelial carcinoma of the bladder. Urol Int. 2011;87(2):143-50.

21. Kramer MW, Merseburger AS, Hennenlotter J, Kuczyk M. Tissue microarrays in clinical urologytechnical considerations. Scand J Urol Nephrol. 2007;41(6):478-84. 
22. Merseburger AS, Hennenlotter J, Schilling D, Sievert KD, Kufer R, Kuczyk MA. Application of tissue microarrays for the diagnosis, prognosis and therapeutic decision making in renal cell carcinoma. Urologe. 2006;45(3):323-4 (326-327).

23. von Wasielewski R, Mengel M, Gignac S, Wilkens L, Werner M, Georgii A. Tyramine amplification technique in routine immunohistochemistry. J Histochem Cytochem. 1997;45(11):1455-9.

24. Mengel M, Werner M, von Wasielewski R. Concentration dependent and adverse effects in immunohistochemistry using the tyramine amplification technique. Histochem J. 1999;31(3):195-200.

25. Mengel M, Kreipe H, von Wasielewski R. Rapid and large-scale transition of new tumor biomarkers to clinical biopsy material by innovative tissue microarray systems. Appl Immunohistochem Mol Morphol. 2003;11(3):261-8.

26. Budczies J, Klauschen F, Sinn BV, et al. Cutoff Finder: a comprehensive and straightforward Web application enabling rapid biomarker cutoff optimization. PLoS One. 2012;7(12):e51862.

27. van Rhijn BW, Burger M, Lotan Y, et al. Recurrence and progression of disease in non-muscle-invasive bladder cancer: from epidemiology to treatment strategy. Eur Urol. 2009;56(3):430-42.

28. Golabek T, Palou J, Rodriguez O, Gaya JM, Breda A, Villavicencio $\mathrm{H}$. Is it possible to stop follow-up of patients with primary T1G3 urothelial carcinoma of the bladder managed with intravesical bacille Calmette-Guerin immunotherapy? World J Urol. 2017;35(2):237-43.

29. Babjuk M, Bohle A, Burger M, et al. EAU guidelines on non-muscle-invasive urothelial carcinoma of the bladder: update 2016. Eur Urol. 2017;71(3):447-61.

30. Parmar MK, Freedman LS, Hargreave TB, Tolley DA. Prognostic factors for recurrence and followup policies in the treatment of superficial bladder cancer: report from the British Medical Research Council Subgroup on Superficial Bladder Cancer (Urological Cancer Working Party). J Urol. 1989;142(2Pt1):284-8.

31. Shariat SF, Zippe C, Ludecke G, et al. Nomograms including nuclear matrix protein 22 for prediction of disease recurrence and progression in patients with Ta, T1 or CIS transitional cell carcinoma of the bladder. J Urol. 2005;173(5):1518-25.

32. Hedegaard J, Lamy P, Nordentoft I, et al. Comprehensive transcriptional analysis of early-stage urothelial carcinoma. Cancer Cell. 2016;30(1):27-42.
33. Abufaraj M, Shariat SF, Foerster B, et al. Accuracy and prognostic value of variant histology and lymphovascular invasion at transurethral resection of bladder. World J Urol. 2018;36(2):231-40.

34. Hermani A, Hess J, De Servi B, et al. Calciumbinding proteins S100A8 and S100A9 as novel diagnostic markers in human prostate cancer. Clin Cancer Res. 2005;11(14):5146-52.

35. Stulik J, Osterreicher J, Koupilova K, et al. The analysis of S100A9 and S100A8 expression in matched sets of macroscopically normal colon mucosa and colorectal carcinoma: the S100A9 and S100A8 positive cells underlie and invade tumor mass. Electrophoresis. 1999;20(4-5):1047-54.

36. Choi JH, Shin NR, Moon HJ, et al. Identification of S100A8 and S100A9 as negative regulators for lymph node metastasis of gastric adenocarcinoma. Histol Histopathol. 2012;27(11):1439-48.

37. Wang W, Ai KX, Yuan Z, Huang XY, Zhang HZ. Different expression of S100A8 in malignant and benign gallbladder diseases. Dig Dis Sci. 2013;58(1):150-62.

38. Tolson JP, Flad T, Gnau V, et al. Differential detection of S100A8 in transitional cell carcinoma of the bladder by pair wise tissue proteomic and immunohistochemical analysis. Proteomics. 2006;6(2):697-708.

39. Yao R, Lopez-Beltran A, Maclennan GT, Montironi R, Eble JN, Cheng L. Expression of S100 protein family members in the pathogenesis of bladder tumors. Anticancer Res. 2007;27(5A):3051-8.

40. Pilchowski R, Stohr R, von Eggeling F, Hartmann A, Wunderlich $\mathrm{H}$, Junker K. Specific protein patterns characterize metastatic potential of advanced bladder cancer. J Urol. 2011;186(2):713-20.

41. Minami S, Sato Y, Matsumoto T, et al. Proteomic study of sera from patients with bladder cancer: usefulness of S100A8 and S100A9 proteins. Cancer Genom Proteom. 2010;7(4):181-9.

42. Ebbing J, Mathia S, Seibert FS, et al. Urinary calprotectin: a new diagnostic marker in urothelial carcinoma of the bladder. World $\mathrm{J}$ Urol. 2014;32(6):1485-92.

43. Kim WJ, Kim SK, Jeong $\mathrm{P}$, et al. A four-gene signature predicts disease progression in muscle invasive bladder cancer. Mol Med. 2011;17(5-6):478-85.

44. Ha YS, Kim MJ, Yoon HY, et al. mRNA expression of S100A8 as a prognostic marker for progression of non-muscle-invasive bladder cancer. Korean J Urol. 2010;51(1):15-20. 
45. Kim SK, Kim EJ, Leem SH, Ha YS, Kim YJ, Kim WJ. Identification of S100A8-correlated genes for prediction of disease progression in non-muscle invasive bladder cancer. BMC Cancer. 2010;10:21.

46. Bansal N, Gupta A, Sankhwar SN, Mahdi AA. Lowand high-grade bladder cancer appraisal via serum- based proteomics approach. Clin Chim Acta. 2014;436:97-103.

47. Bansal N, Gupta AK, Gupta A, Sankhwar SN, Mahdi AA. Serum-based protein biomarkers of bladder cancer: a pre- and post-operative evaluation. J Pharm Biomed Anal. 2016;124:22-5. 